\title{
Hydrogen-Bond Dynamics in the Air-Water Interface
}

\author{
Pu Liu, Edward Harder, and B. J. Berne* \\ Department of Chemistry and Center for Bimolecular Simulation, Columbia University, 3000 Broadway, \\ New York, New York 10027
}

Received: July 19, 2004; In Final Form: November 15, 2004

\begin{abstract}
Hydrogen-bond (H-bond) dynamics in the air-water interface is studied by molecular dynamics simulations. The analysis reveals that the dynamics of breaking and forming hydrogen bonds in the air-water interface is faster than that in bulk water for the polarizable water models. This is in contrast to the results found on a protein surface. We show that the difference stems from more rapid translational diffusion in the interface. When the effect of pair diffusion is eliminated, the hydrogen-bond dynamics in the interface is observed to be slower than that in the bulk. This occurs because the number of water molecules adjacent to a hydrogenbonded pair and available to accept or donate a hydrogen bond is smaller in the interface than in the bulk. The comparison between polarizable water models and fixed-charge models highlights the potential importance of the polarization effect in the water-vapor interface.
\end{abstract}

\section{Introduction}

The study of liquid interfaces has applications to a wide array of chemical and physical processes. Such processes include phase-transfer catalysis ${ }^{1}$ and drug delivery in pharmacology. ${ }^{2}$ In particular, understanding the air-water interface is essential to elucidating the uptake of pollutants across the liquid-vapor interface in atmospheric chemistry. ${ }^{3}$ The asymmetrical environment of the air-water interface provides a solvent region with properties distinct from those of bulk water. Using traditional spectroscopic techniques to study interfaces is a challenging task because of the signal dominance of the bulk phase. Techniques such as second-harmonic generation and sum-frequency generation can selectively probe interface regions, thus circumventing this problem. ${ }^{4}$ Recently, magnetic relaxation dispersion (MRD) has been used to study the dynamics of protein hydration directly. ${ }^{46}$ Another option is computer simulation. Simulations provide atomic detail and are thus ideal tools for studying the air-water interface. ${ }^{5}$

Understanding hydrogen bonds is the key to exploring the peculiar properties of water ${ }^{6}$ and aqueous systems. Over the past two decades, considerable work has been done on the study of the hydrogen-bond behavior of water. ${ }^{7-10,12-16}$ However, the previous studies have focused mainly on bulk water or solutes dissolved in bulk water. Some work has been done on hydrogenbond dynamics at vapor-liquid interfaces. ${ }^{41}$ We set out to study the dynamics of hydrogen bonds in the air-water interface using molecular dynamics simulations and a polarizable water model, TIP4P/FQ. ${ }^{17}$ We expected to observe hydrogen-bond behavior similar to that found on the nonpolar surface of proteins ${ }^{15,18}$ because the vapor phase is essentially nonpolar (low dielectirc constant) and the orientational distribution of water molecules next to hydrophobic surfaces is similar to that of water molecules in the liquid-vapor interface. ${ }^{16,19}$

The formation and breaking of water-water hydrogen bonds are highly concerted processes,${ }^{18}$ and the relaxation rate of these processes increases as the number of adjacent but non-hydrogen-

* To whom correspondence should be addressed. E-mail: berne@ chem.columbia.edu. bonded water molecules increases. Because the number of such "replacement" water molecules in the interfacial region is smaller than that in the bulk, one might expect the hydrogenbond dynamics to be slower. However, our simulation results show that the hydrogen-bond dynamics in the air-water interface speeds up. Hydrogen bonds can break either by relative translational diffusion or by reorientation. In a previous work, ${ }^{40}$ we showed that translational diffusion speeds up in the interface. We find that the hydrogen-bond dynamics, with the effects of pair-translational diffusion removed, actually slows down in the interface. The above-mentioned speedup can be atributed to the speedup of translational diffusion in the interface.

This paper is organized as follows. The following section outlines details of the molecular dynamics simulations. The results for the hydrogen-bond autocorrelation function and the diffusion coefficients for the bulk and air-water interface are given in section III. In section III, the comparison between polarizable water models and fixed-charge models is also presented.

\section{Simulation Method}

Because the molecular electronic structure can be greatly affected by the environment, the dynamical fluctuating-charge force field is a more suitable candidate than other fixed-charge force fields, such as TIP4P ${ }^{20}$ or SPC, ${ }^{21}$ in studying two-phase equilibrated systems. For example, the total dipole moment of water in the liquid phase is $2.5 \mathrm{D}$, whereas it is $1.85 \mathrm{D}$ in the gas phase. The charge used in fixed-charge models cannot be used for different phases because the charge magnitudes reflect the value for one specific phase. The study of water-water hydrogen-bond dynamics in particular has been shown to be sensitive to water polarizability, and its inclusion in the simulation should not be ignored. ${ }^{14}$ We thus use the TIP4P/FQ water model. ${ }^{17,20}$ The TIP4P/FQ water model has the same geometry as the TIP4P water model, but the partial charges can fluctuate in response to environmental changes. For comparison, a new five-site water model, POL5, ${ }^{44}$ is chosen. In the meantime, the TIP4P 43 water model and $\mathrm{SPC} / \mathrm{E}^{42}$ are selected as the representatives for fixed-charge models. 
To use larger time steps in the simulation, we froze molecular vibrations by keeping bond lengths at their equilibrium values. The coordinate correction algorithm, RATTLE, ${ }^{22}$ is used to constrain the bond lengths, allowing for a time step of $1 \mathrm{fs}$ in our simulations. We use 3D periodic boundary conditions. The mesh-based approximations to the Ewald sum ${ }^{23}$ can be used to calculate the electrostatic interactions efficiently. ${ }^{24-27}$ We use the $\mathrm{P} 3 \mathrm{ME}^{24}$ method. We choose a real-space cutoff of 11.25 $\AA$. The corresponding Ewald splitting parameter is $0.376 \AA^{-1}$. The choice of an isotropic Ewald splitting parameter requires that the density of $k$-space vectors remains the same in each Cartesian coordinate. ${ }^{28}$ For the P3ME method, this translates to a constant density of grid points in each coordinate. The gridpoint density we use is $0.5 \AA$.

Our liquid-vapor system is constructed in the following manner. One cubic box of 512 water molecules is equilibrated for $200 \mathrm{ps}$ with $1 \mathrm{~atm}$ and $T=298.15 \mathrm{~K}$ in an NPT ensemble, using an Anderson-Hoover barostat ${ }^{29}$ and a Nose-Hoover chain thermostat ${ }^{30}$ to determine the initial box length of $25 \AA$. The original system of interest is periodic in two dimensions (chosen as the $x$ and $y$ coordinates) and nonperiodic in the $z$ coordinate perpendicular to the interfacial surface. Using Ewald summation for a 2D system is considerably more costly than the standard 3D formulation. ${ }^{31}$ If sufficient empty space is allowed for the vapor phase in constructing the water layer, then the error incurred by using a 3D Ewald formula for a 2D system becomes negligible for a charge-neutral system. The space allotted to the vapor should be as large as the box lengths perpendicular to the interface normal, $L_{x}$ and $L_{y} .{ }^{31} \mathrm{We}$ add 25 $\AA$ in the $z$ coordinate to both sides of our resultant box from the NPT equilibration. Yeh and Berkowitz ${ }^{32}$ have also noted that an additional shape-dependent term is necessarily added to the force contribution for systems with a nonzero component of the total dipole moment in the $z$ direction. Because of the symmetry of our system, the $z$ component of the dipole moment is zero, and this term can be ignored. The dimensions of our system are thus $L_{x}=25 \AA, L_{y}=25 \AA$, and $L_{z}=75 \AA$. The rectangular system is then equilibrated for another $500 \mathrm{ps}$ in an NVT ensemble at $T=298.15 \mathrm{~K}$ using the Nose-Hoover integration algorithm. ${ }^{33}$

The result is a system with two air-water interfaces that are perpendicular to the $z$ direction. For the TIP4P/FQ water model, nine independent configurations sampled from the NVT ensemble are employed as the initial phase points to conduct the following simulations in the NVE ensemble with the velocity Verlet integrator. For each simulation, data collection begins by recording the trajectory every 100 fs for another 200 ps. Furthermore, for each model, the last configuration from the 500-ps NVT simulation is used as the starting point. After the 150-ps NVT equilibration stage, the configurations are recorded every 40 fs for another 400-ps NVE simulation. Three independent trajectories at three defferent temperatures, 280, 298, and $330 \mathrm{~K}$, are generated for each model. All of the calculations were performed using SIM, the molecular dynamics program developed in the Berne group. ${ }^{34}$

\section{Results and Discussion}

It is necessary to characterize the system appropriately once equilibrium has been reached for the liquid-vapor system. A density profile along the $z$ axis is calculated to illustrate the structural change from bulk water to the interface. The profile is constructed by slicing the simulation cell into $1-\AA$ partitions along the $z$ axis and calculating the density within each of these spatial slices. The density profile corresponding to the produc-

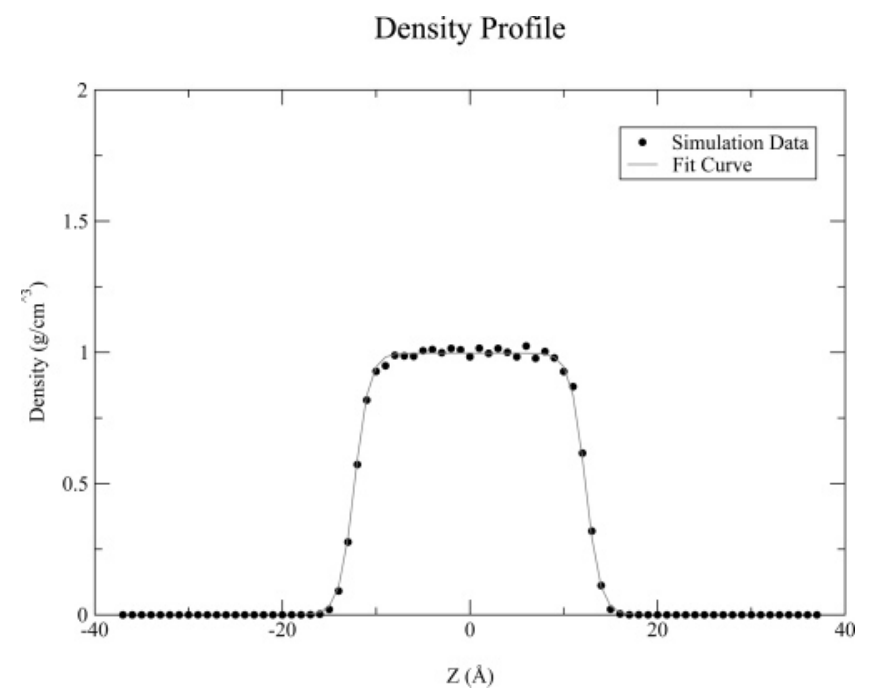

Figure 1. Density profile of 512 water molecules at $298.15 \mathrm{~K}$ along the interface normal for the TIP4P/FQ water model. The simulation box has dimensions of $L_{x}=25 \AA, L_{y}=25 \AA$, and $L_{z}=75 \AA$. The profile is constructed by slicing the simulation cell into 1-A partitions along the $z$ axis and calculating the density within each of these spatial slices. The solid line is the fitted curve using the hyperbolic tangent function described in section III.

tion run is shown in Figure 1. The profile shows a plateau region with a density corresponding to the bulk water phase centered about the origin of the simulation cell. Two interface regions are also evident approximately $\pm 12.5 \AA$ from the center of the box, and the vapor phase corresponds to the regions of nearzero density. A hyperbolic tangent function can be fit to the density profile: ${ }^{35}$

$$
\rho(z)=\frac{1}{2}\left(\rho_{\mathrm{L}}+\rho_{\mathrm{V}}\right)-\frac{1}{2}\left(\rho_{\mathrm{L}}-\rho_{\mathrm{V}}\right) \tanh \left[\frac{\left(z-z_{0}\right)}{d}\right]
$$

The width of our interface region is chosen as the "10-90" thickness. ${ }^{36}$ This thickness describes the density change from 90 to $10 \%$ of the bulk water density. The width for our system is approximately $3.5 \AA$ for the TIP4P/FQ water model. The density profiles for other water models are just slightly different from those of the TIP4P/FQ water model. (The results are not presented here.)

An appropriate definition for a hydrogen bond is necessary to study the behavior of hydrogen bonds in the interface. Two such definitions are usually applied. The first is defined by an interaction energy between two water molecules more negative than $-10 \mathrm{~kJ} / \mathrm{mol} .{ }^{35}$ The second definition is based on the relative geometry between a pair of water molecules. This definition requires the oxygen-oxygen distance to be less than $3.5 \AA$ and the $\mathrm{H}-\mathrm{O} \cdots \mathrm{O}$ angle to be less than $30^{\circ}$. We will employ the geometric definition because this choice has been shown to provide more reliable results in simulations. ${ }^{37}$

The top panel of Figure 2 shows the average number of $\mathrm{H}$ bonds per water molecule found in each slice of our simulation cell. As expected, the number of $\mathrm{H}$ bonds falls off with the density of water molecules in each slice. A more insightful figure, the bottom panel of Figure 2, is a plot of the number of $\mathrm{H}$ bonds in each slice divided by the coordination number for water. The coordination number is the number of water molecules within $3.5 \AA$ of a tagged molecule. This quantity reflects the probability of a hydrogen bond existing between coordinated waters. Beyond the interface, few hydrogen bonds can be formed because of the extremely low density. Our results show a larger probability for hydrogen bonding on the surface 

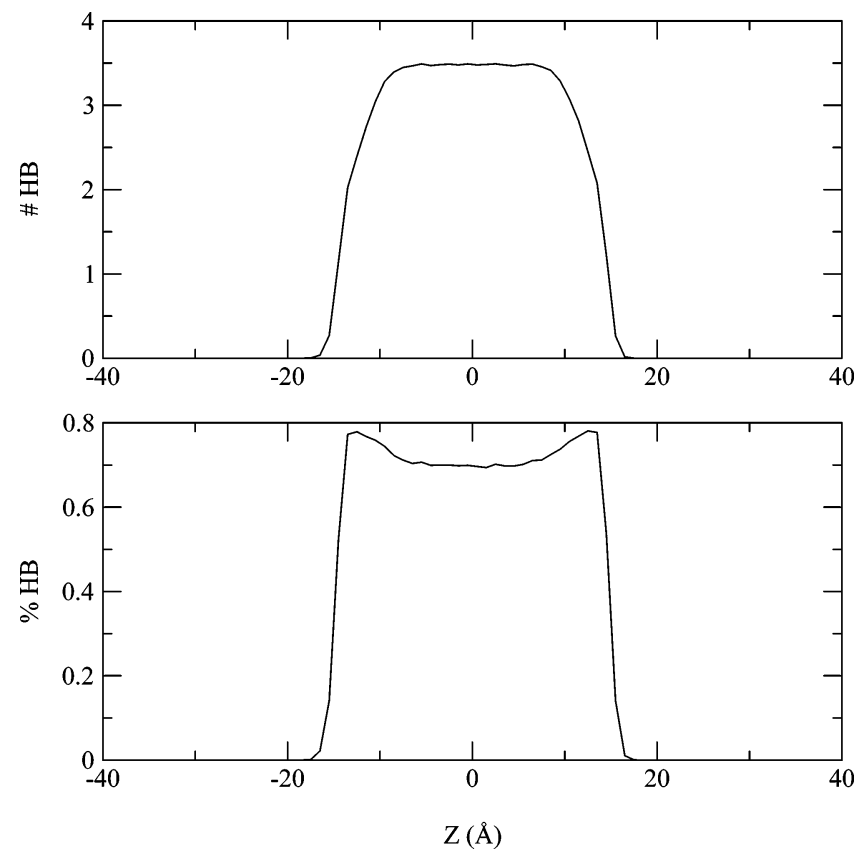

Figure 2. Top panel: Number of $\mathrm{H}$ bonds per water molecule vs the location along the interface normal for the TIP4P/FQ water model. The $\mathrm{H}$ bond is defined by an oxygen-oxygen distance less than $3.5 \AA$ and an $\mathrm{H}-\mathrm{O} \cdots \mathrm{O}$ angle less than $30^{\circ}$. Bottom panel: Number of $\mathrm{H}$ bonds per water molecular divided by the coordination number of the water molecules. The coordination number is the number of water molecules within $3.5 \AA ̊$ of a tagged molecule.

than that in the bulk. Similar results have been found elsewhere. ${ }^{35,38}$ The inference from these studies is that although there are fewer hydrogen bonds in the interface the water molecules have a stronger tendency to be hydrogen bonded with each other. ${ }^{35}$

We will study hydrogen-bond kinetics ${ }^{10}$ on the interface to develop a more robust understanding of the dynamical behavior of interfacial $\mathrm{H}$ bonds. The $\mathrm{H}$-bond autocorrelation function is

$$
c(t)=\frac{\langle h(0) h(t)\rangle}{\langle h\rangle}
$$

where $h(t)$ is the H-bond population operator and has a value of 1 if a $\mathrm{H}$ bond is present at time $t$ or zero if a $\mathrm{H}$ bond is not present. The function $\langle h\rangle$ is the average of $h(t)$. The autocorrelation function measures the probability that a $\mathrm{H}$ bond is present at time $t$ given the presence of the $\mathrm{H}$ bond at time zero, essentially measuring the structural relaxation of $\mathrm{H}$ bonds. The correlation function can be further classified into historydependent and history-independent correlations. ${ }^{8,37}$ The historydependent definition measures bond autocorrelations for bonds that remain continuously unbroken. We use the historyindependent definition that measures bond autocorrelations including bonds that have broken and reformed.

We will calculate $c(t)$ in the interface, denoted $c_{\text {interface, }}$ and compare this with $c(t)$ found in the bulk, denoted $c_{\text {bulk }}(t)$. The $\mathrm{H}$ bonds included in the calculation of $c_{\text {interface }}(t)$ are those for which the pair of water molecules are within the 10-90 surface $^{36}$ at $t=0$. The $c_{\text {bulk }}(t)$ will include hydrogen bonds between water molecules, both of which are found in a $3.5-\AA$ region centered about the origin. The results are given in Figure 3 We can calculate the associated relaxation time for the $\mathrm{H}$ bonds using the relation $c\left(\tau_{\mathrm{rlx}}\right)=\mathrm{e}^{-1,37,45}$ from which the relative time scale can be obtained. The relaxation times are $\tau_{\mathrm{rlx}}^{\text {bulk }}=$ 4.93 ps and $\tau_{\mathrm{rlx}}^{\text {interface }}=4.07$ ps.

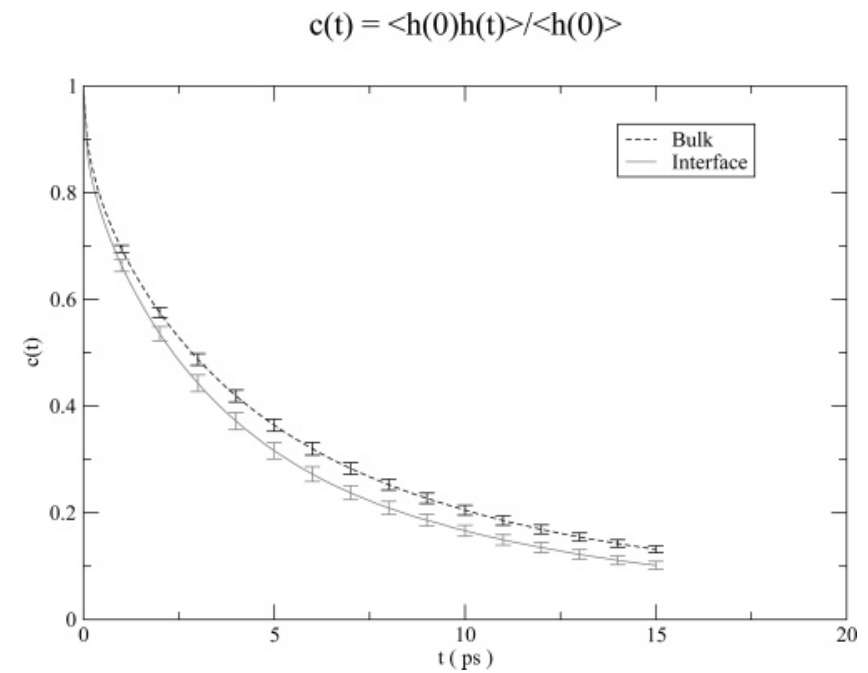

Figure 3. Hydrogen-bond autocorrelation function $c(t)$ of the interface and the bulk for the TIP4P/FQ water model. We use the historyindependent definition for a $\mathrm{H}$ bond, which includes the contribution from $\mathrm{H}$ bonds that have broken and reformed. The result presented is the average of nine trajectories.

$$
\mathrm{O}(\mathrm{t})=\langle\mathrm{h}(0) \mathrm{h}(\mathrm{t}) \mathrm{H}(\mathrm{t})>/<\mathrm{h}(0) \mathrm{H}(\mathrm{t})>
$$

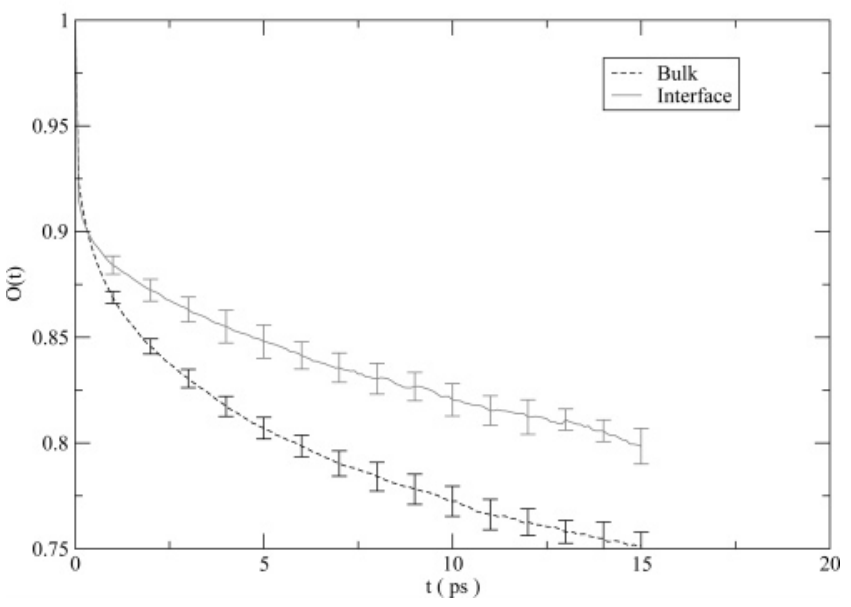

Figure 4. Conditional hydrogen-bond autocorrelation after eliminating the effect of diffusion for the bulk and the interface for the TIP4P/FQ water model. This is done by using an operator $H(t)$, defined as 1 if the water pair is within $3.5 \AA$ and defined as zero otherwise. The result presented is the average of nine trajectories.

The relative relaxation times make sense when one considers the effects of diffusion. We calculated the diffusion coefficients for the bulk and interfacial regions. The Einstein relation is normally used to calculate self-diffusion coefficients from simulations $^{39}$

$$
\left\langle\Delta r(t)^{2}\right\rangle \rightarrow 2 d D t
$$

where $d$ is the number of translational degrees of freedom. However, the relation is derived assuming a homogeneous system and thus is not suitable for our inhomogeneous airwater interface system. Instead, we developed a general methodology to evaluate the diffusion coefficients in an inhomogeneous liquid or a confined liquid. ${ }^{40}$ The diffusion coefficient in the interface can be reduced to a component parallel to the surface $D_{x x}=D_{y y}=0.8 \AA^{2} / \mathrm{ps}(f=2)$ and a component perpendicular to the surface $D_{z}=0.5 \AA^{2} / \mathrm{ps}(f=$ 1), ${ }^{40}$ as seen in Figure 5. For the bulklike region, $D_{x x}=D_{y y}=$ $D_{z z}=0.22 \AA^{2} / \mathrm{ps}$, which is similar to the experimental result of 


\section{z-dependance of Diffusion Coefficient}

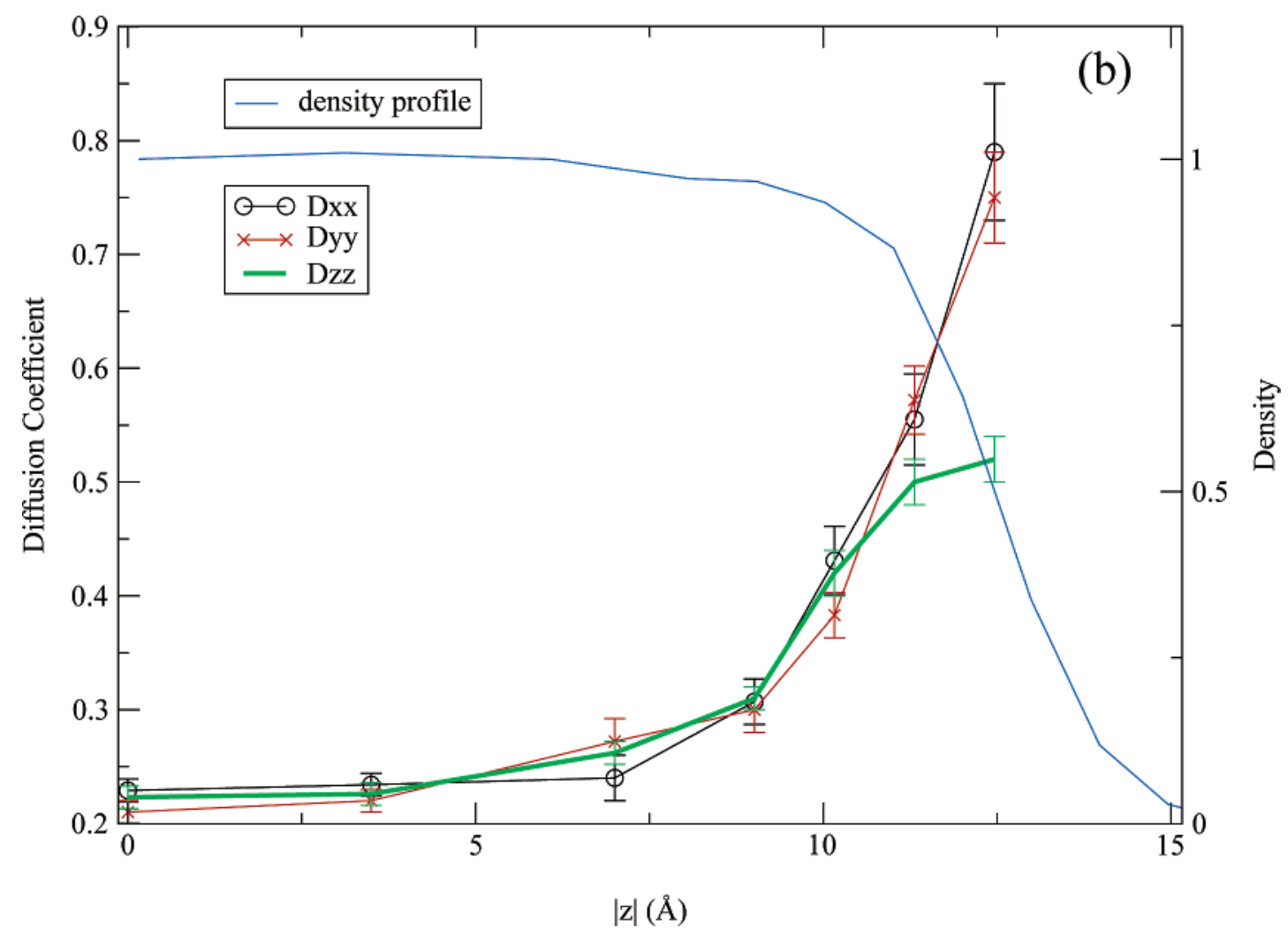

Figure 5. Plot of the diffusion coefficient in all three Cartesian coordinates for 3.5- $\AA$ regions extending from the bulk up to the interface for the TIP4P/FQ water model.

$0.23 \AA^{2} / \mathrm{ps} .{ }^{47}$ As one might expect, the drop in density in the interface reduces steric constraints in translational motion and increases the diffusion coefficient on the interface relative to that of the bulk. Luzar and Chandler have shown that H-bond kinetics for times longer than 1 ps is related to pair diffusion. ${ }^{10}$ Therefore, the faster relaxation time in the interface can be attributed to the larger diffusion coefficient in this region. To eliminate the effects of diffusion, we calculate the following correlation function

$$
O(t)=\frac{\langle h(0) h(t) H(t)\rangle}{\langle h(0) H(t)\rangle}
$$

where the operator $H(t)=1$ if the water pair is within $3.5 \AA$ and $H(t)=0$ otherwise. $O(t)$ is the conditional probability of a hydrogen bond being present at time $t$, given that it was present at time $t=0$, with the added constraint that the pair has not diffused away from each other. This results in an inversion in the qualitative relaxation behavior of the two regions. From Figure 4, we find that the correlation function for the interfacial region decays more slowly than that for the bulk region when the effects of diffusion are removed. This result can be explained by a concerted mechanism for $\mathrm{H}$-bond dynamics. H-bond breaking can be completed only if the participating water molecules can form new $\mathrm{H}$ bonds with neighboring molecules. If no non-H-bonded neighbors are available to a bonded pair, then they will stay bonded for the most part. Thus, the dynamic behavior of individual $\mathrm{H}$ bonds will depend on $n_{\text {adj }}$, the number of adjacent water molecules not presently $\mathrm{H}$-bonded and thus available for bonding. The quantity $n_{\mathrm{adj}}$ has been shown to depend significantly on the environment. ${ }^{9,15}$ Figure 6 shows the distribution of $n_{\text {adj }}$ for the bulk and interfacial regions. The

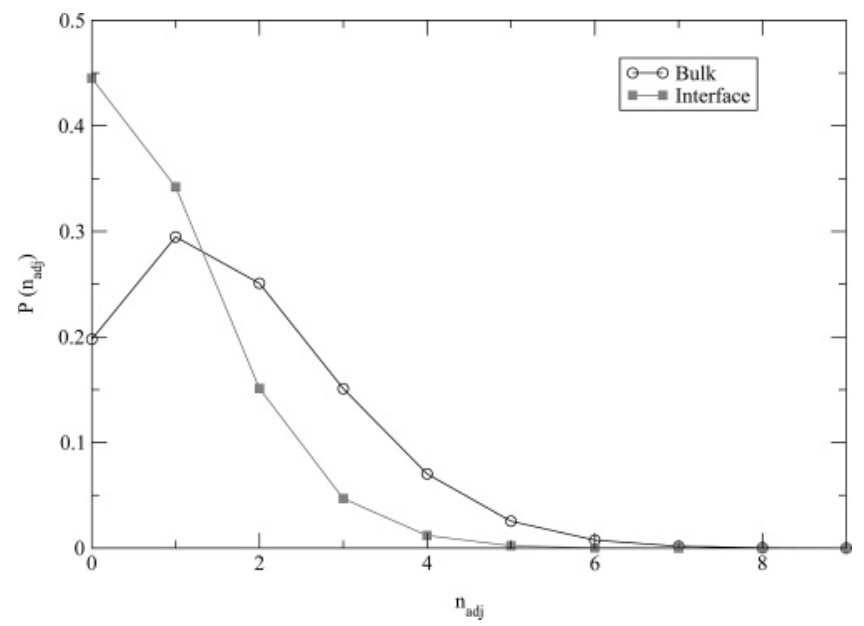

Figure 6. Distribution of the number of adjacent but non-hydrogenbonded water molecules for the bulk region and the interfacial region for the TIP4P/FQ water model.

second panel of Figure 2, showing the percentage of $\mathrm{H}$ bonds in the coordination shell as a function of proximity to the interface, gives similar information. In the interfacial region, it can be seen that a larger percentage of neighboring water molecules are hydrogen bonded and thus $n_{\text {adj }}$ is smaller. Clearly in our case, symmetry breaking caused by the interface reduces $n_{\text {adj }}$ as compared to that of bulk water. This explains the inversion in relaxation times when the effect of pair diffusion is removed from the calculation of the H-bond autocorrelations.

To illustrate the effect of the diffusion on the hydrogen-bond dynamics, we adopted the method of Luzar and Chandler. By applying the simple model for hydrogen-bond kinetics, the 


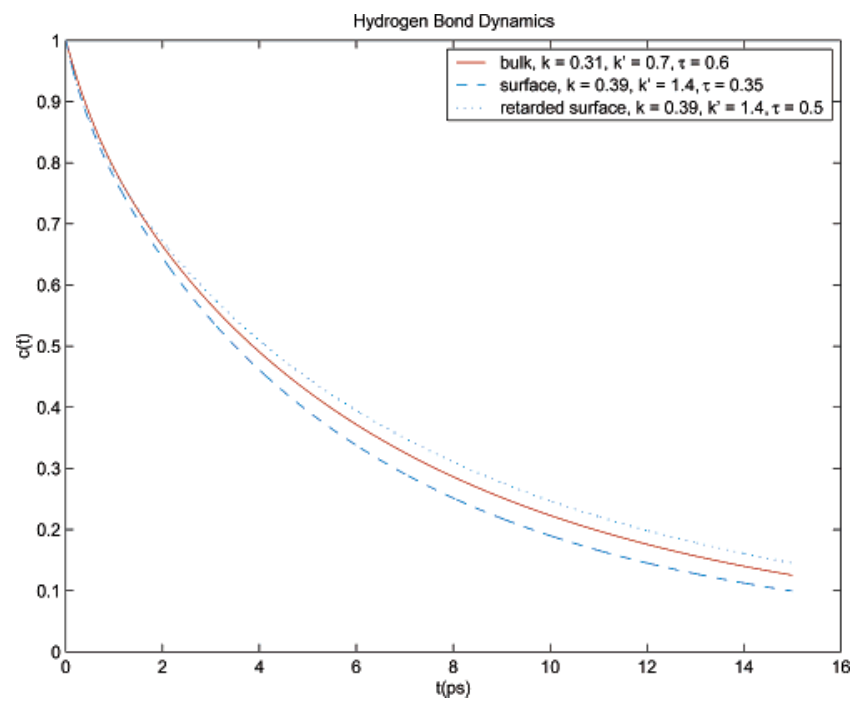

Figure 7. Hydrogen-bond dynamics calculated from the numerical integration of the rate of hydrogen-bond dynamics relaxation, $k(t)=$ $-\mathrm{d} c(t) / \mathrm{d} t . k(t)$ is the inverse Laplace transform of $\tilde{k}(s)=k /[s+k+$ $\left.k^{\prime} s f(s)\right]$, where $f(s)=3 \tau[1-\sqrt{s \tau} \arctan (1 / \sqrt{s \tau})] .{ }^{10}$ The red solid line, the blue long-dashed line, and the blue short-dashed line are for the bulk, the interface, and the retarded surface, respectively. The corresponding parameters, $k, k^{\prime}$, and $\tau$, are listed in the legend for each case.

forward and backward rate constants, $k$ and $k^{\prime}$, can be calculated from the equation

$$
-\frac{\mathrm{d} c(t)}{\mathrm{d} t}=k c(t)-k^{\prime} n(t)
$$

where $n(t)$ is the probability that the hydrogen bond is broken
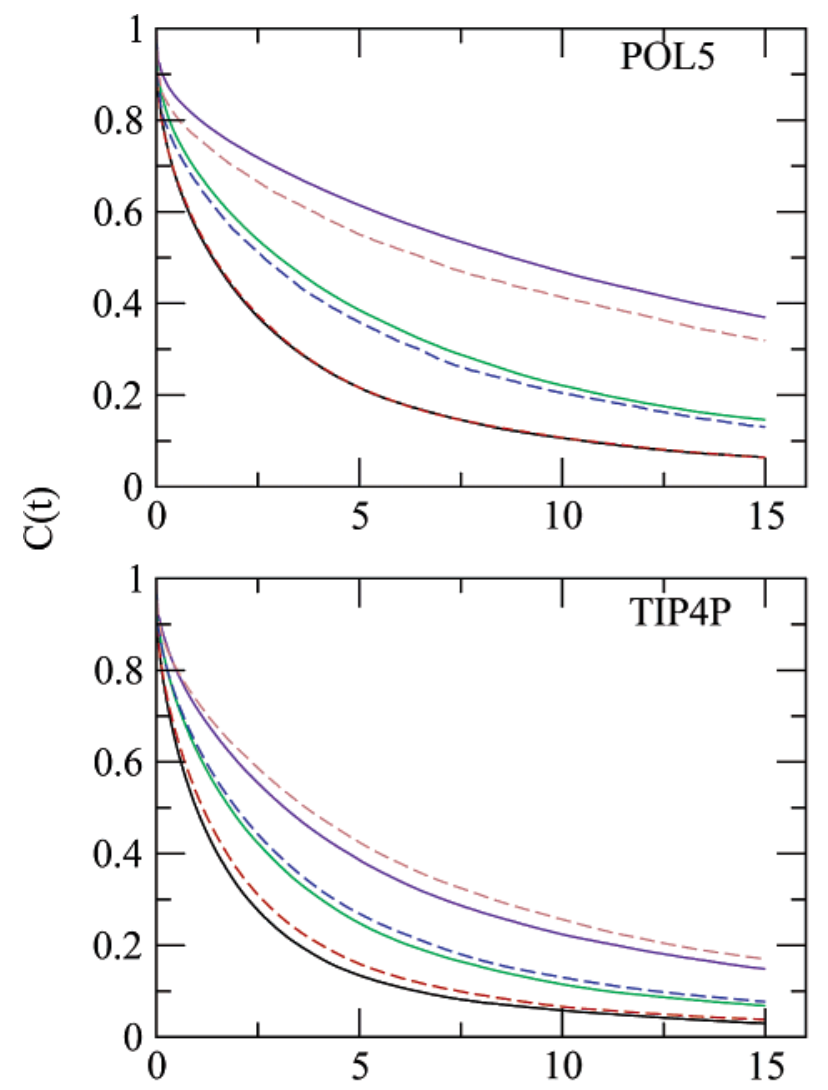

at time $t$ but this pair of water molecules has not diffused away. For the bulk region, the $k$ and $k^{\prime}$ are best fitted to be 0.31 and $0.71 \mathrm{ps}^{-1}$, respectively, which are very close to the results reported by $\mathrm{Xu}$ and Berne. ${ }^{14}$ For the interface region, $k$ and $k^{\prime}$ are 0.39 and $1.4 \mathrm{ps}^{-1}$, respectively. To solve the diffusion system with source and sink terms, eqs 11 and 12 in ref 11 , we introduced the parameter $\tau\left(\tau=a^{2} / D\left(6 \pi^{2}\right)^{2 / 3}\right)$ for the regularization of spatial resolution, where $a$ is the distant a bonded pair that can move without breaking the bond. It is estimated to be less than 1 ps. ${ }^{10,11}$ For our result, $\tau$ is chosen to be $0.6 \mathrm{ps}$ for the bulk to fit our simulation result. Because the pair diffusion in the interface is much faster than that in the bulk, the $\tau$ for the interface is smaller. Similarly, it is chosen as 0.35 ps. If you substitute those parameters into the Laplace transform of $k(t)^{10}$ and do the inverse Laplace transform, then $k(t)(k(t)=$ $-\mathrm{d} c(t) / \mathrm{d} t)$ and $c(t)$ can be obtained. Figure 7 shows the numerical results. Clearly, the hydrogen-bond dynamics for the interface is faster than that for the bulk. However, if the pair diffusion is retarded in the interface, for example, if $\tau$ is increased from 0.35 to $0.5 \mathrm{ps}$, then the relative behavior of the hydrogen-bond dynamics can be inverted.

A recent publication addressing the same problem with the fixed-charge model $\mathrm{SPCE}^{42}$ shows that the hydrogen-bond dynamics is slower in the interface than in the bulk, in contrast to our current result. ${ }^{41}$ To analyze the difference in the hydrogenbond relaxation on the liquid-vapor interface between fixedcharge water models and polarizable models further, we performed a series of simulations for two kinds of polarizable models, TIP4P/FQ and POL5, ${ }^{44}$ and two types of fixed-charge models, TIP4P ${ }^{43}$ and SPC/E. ${ }^{42}$ Consistent results were found. For polarizable models, the dynamics in the interface is usually faster than that in the bulk region. For the fixed-charge models,
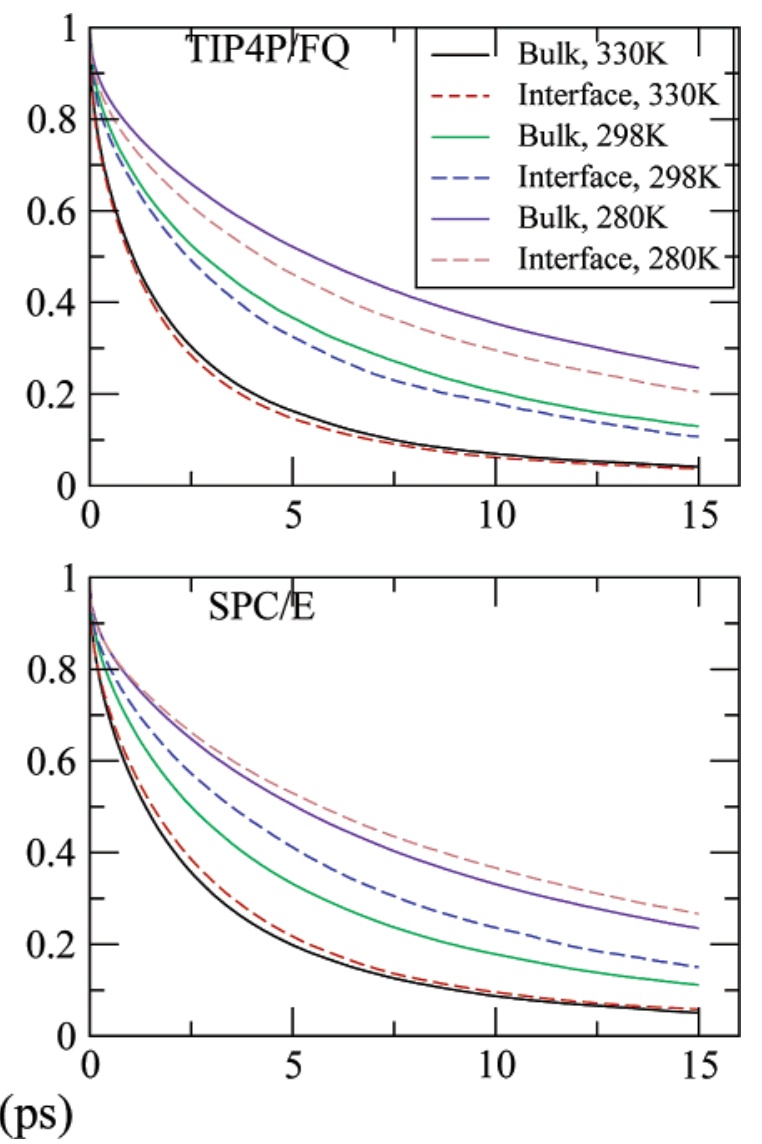

Figure 8. Hydrogen-bond dynamics for different water models and different temperatures. The models and the simulated temperatures are indicated in the legends. 

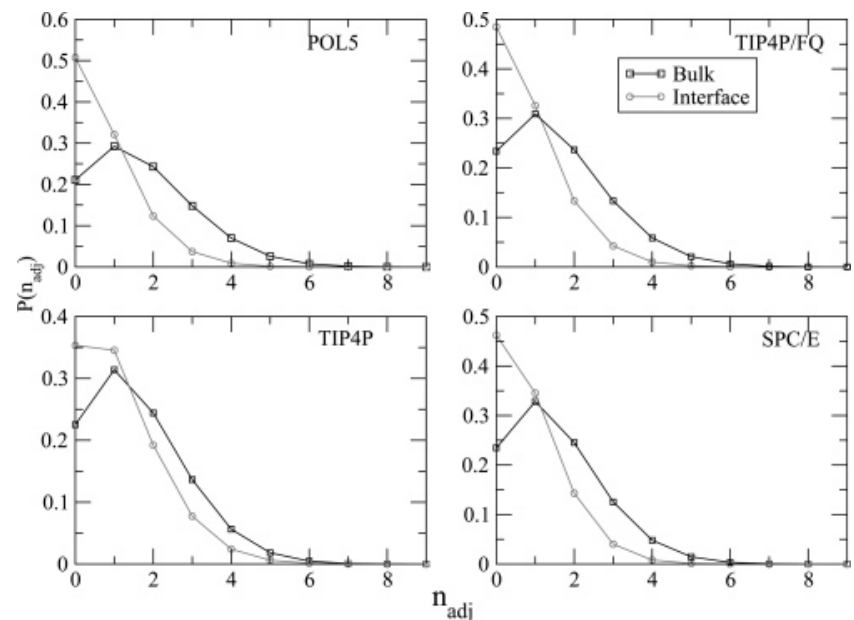

Figure 9. Distribution of the number of adjacent but non-hydrogenbonded water molecules for the bulk region and the interfacial region for different water models at $T=298 \mathrm{~K}$. The models used are indicated in the legends.

however, it is always slower in the interface than in the bulk. To understand the difference, we examined the relative behavior of hydrogen-bond dynamics without the effect of diffusion, $O(t)$. For all of the water models and all of the temperatures we studied, the function $O(t)$ relaxes slower in the interface than in the bulk. (The results for $O(t)$ are not presented here.) The function $n_{\mathrm{adj}}$, a good representative of the structural factor in hydrogen-bond dynamics, is similar between different models, which is shown in Figure 9. This implies that the difference in pair diffusion between the bulk and the interface is smaller for the fixed-charge models compared with that of the polarizable models. For the polarizable water models, the effects of polarization enhance the interaction of pairs of water molecules in the liquid phase mutually, which will slow the dynamics of the liquid phase. This is clearly shown in Figure 8. However, in the vapor-liquid interface, the environment is quite different from that in the bulk phase. Because of smaller polarization, the interaction of water pairs in the interface is weaker than that in the bulk for polarizable water models. For the fixedcharge model, the partial charges are parametrized for the liquid phase and are usually overestimated for the liquid-vapor interface. This factor will accelerate the hydrogen-bond dynamics in the interface for polarizable models and slow it down for the fixed-charge models. This appears to be the physical explanation of the difference between polarizable water models and fixed-charge models.

\section{Conclusions}

This paper explores the hydrogen-bond dynamics in the liquid-vapor interface of water. It is found that as a water molecule nears the interface the relaxation of hydrogen bonds becomes faster, corresponding to an increase in the diffusion coefficient in the interfacial region (Figure 5). The rate of hydrogen-bond relaxation arises from an interplay between the relative translational diffusion of an initially hydrogen-bonded pair and the $\mathrm{H}$-bond making and breaking, which is governed by the relative orientation of this $\mathrm{H}$-bond pair. ${ }^{10}$ Translational diffusion determines whether a specific pair of water molecules are still adjacent to each other after some amount of time. Because the diffusion coefficient increases as a water molecule nears the interfacial region, the rate of hydrogen-bond relaxation for the interfacial water molecules will increase. However, the asymmetric geometry of the interface decreases the number of non-hydrogen-bonded but coordinated water pairs. Thus, the breaking and forming of hydrogen bonds will be hampered in the interface region because the H-bond breaking will be more likely to happen if the participating water molecules can form new $\mathrm{H}$ bonds with neighboring molecules. For the air-water interface system, the rate of hydrogen-bond relaxation increases as a water molecule nears the interface, clearly showing that the role played by diffusion becomes more and more prominent when a water molecule nears the interface. Water-solid interfaces, such as protein surfaces, where the overall hydrogen dynamics is slower than that in the bulk water, ${ }^{15}$ may provide an intriguing system in which the translational pair diffusion is not the dominant factor in hydrogen-bond dynamics.

Acknowledgment. This work has been supported by a grant for B.J.B. from the NSF (CHE-03-16896).

\section{References and Notes}

(1) Starks, C.; Liotta, C.; Halpern, M. Phase Transfer Catalysis; Chapman and Hall: New York, 1994.

(2) Arai, K.; Ohsawa, M.; Kusu, F.; Takamura, K. Bioelectrochem. Bioenerg. 1993, 31, 65.

(3) Gennis, R. Biomembranes Springer: New York, 1989

(4) Eisenthal, K. Acc. Chem. Res. 1993, 26, 636.

(5) Benjamin, I. Modern Methods for Multidimensional Dynamics Computations in Chemistry; World Scientific: Singapore, 1998. 1981.

(6) Angell, C. Water: A Comprehensive Treatise; Plenum: New York,

(7) Stillinger, F. Science 1980, 209, 451.

(8) Sciortino, F.; Poole, P.; Stanley, H.; Havlin, S. Phys. Rev. Lett. 1990, 64, 1686

(9) Luzar, A.; Chandler, D. Phys. Rev. Lett. 1996, 76, 928.

(10) Luzar, A.; Chandler, D. Nature 1996, 379, 55.

(11) Luzar, A. J. Chem. Phys. 2000, 113, 10663.

(12) Starr, F.; Nielsen, J.; Stanley, H. Phys. Rev. Lett. 1999, 82, 2294

(13) Starr, F.; Nielsen, J.; Stanley, H. Phys. Rev. E: Stat. Phys., Plasmas, Fluids, Relat. Interdiscip. Top. 2000, 62, 579.

(14) Xu, H.; Berne, B. J. Phys. Chem. B 2002, 106, 2054.

(15) Xu, H.; Berne, B. J. Phys. Chem. B 2001, 105, 11929

(16) Taylor, R.; Dang, L.; Garrett, B. J. Phys. Chem. 1996, 100, 11720.

(17) Rick, S.; Stuart, S.; Berne, B. J. Chem. Phys. 1994, 101, 6141.

(18) Luzar, A. Faraday Discuss. 1996, 103, 29.

(19) Huang, X.; Margulis, C.; Berne, B. J. Phys. Chem. B 2003, 107, 11742

(20) Jorgensen, W.; Chandrasekhar, J.; Madura, J.; Impey, R.; Klein, M. J. Chem. Phys. 1983, 79, 926.

(21) Berendsen, H.; Postma, J.; von Gunsteren, W.; Hermans, J. Intermolecular Forces; Reidel: Dordrecht, The Netherlands, 1981.

(22) Andersen, H. J. Comput. Phys 1983, 52, 24.

(23) Ewald, P. Ann. Phys. (Berlin) 1921, 64, 253.

(24) Zhou, R.; Harder, E.; Xu, H.; Berne, B. J. Chem. Phys. 2001, 115, 2348 .

(25) Darden, T.; York, D.; Pedersen, L. J. Chem. Phys. 1993, 98, 10089

(26) Luty, B. A.; Tironi, I. G.; van Gunsteren, W. F. J. Chem. Phys. 1995, $103(8), 3014$.

(27) Hockney, R. W.; Eastwood, J. W. Computer Simulation Using Particles; IOP: Bristol, England, 1988.

(28) Alejandre, J.; Tildesley, D.; Chapela, G. J. Chem. Phys. 1995, 102 4574

(29) Martyna, G.; Tobias, D.; Klein, M. J. Chem. Phys. 1994, 101, 4177.

(30) Martyna, G.; Klein, M.; Tuckerman, M. J. Chem. Phys. 1992, 97 , 2635 .

(31) Spohr, E. J. Chem. Phys. 1997, 107, 6342.

(32) Yeh, I.; Berkowitz, M. J. Chem. Phys. 1999, 111, 3155.

(33) Hoover, W. Phys. Rev. A 1985, 31, 1695.

(34) Stern, H.; Rittner, F.; Pavese, M.; Harder, E.; Xu, H.; Kim, B. SIM: Molecular Dynamics Simulation Program; Columbia University: New York, NY, 2001.

(35) Benjamin, I. Chem. Rev. 1996, 96, 1449.

(36) Beaglehole, D. Fluid Interfacial Phenomena; Wiley: New York, 1986.

(37) Starr, F.; Nielsen, J.; Stanley, H. Phys. Rev. B 2000, 62, 579

(38) Linse, P. J. Chem. Phys. 1987, 86, 4177.

(39) Allen, M.; Tildesley, D. Computer Simulation of Liquids; Oxford University Press Inc.: New York, 1987.

(40) Liu, P.; Harder, E.; Berne, B. J. Phys. Chem. B 2004, 108, 6595.

(41) Paul, S.; Chandra, A. Chem. Phys. Lett. 2004, 386, 218.

(42) Berendsen, H. J. C.; Grigera, J. R.; Straatsma, T. P. J. Phys. Chem

1987, 91, 6269 . 
(43) Jorgensen, W. L.; Madura, J. D. Mol. Phys. 1985, 56, 1381.

(44) Stern, H. A.; Rittner, F.; Berne, B. J.; Friesner, R. A. J. Chem. Phys. 2001, 115, 2237.

(45) Chandra, A. Phys. Rev. Lett. 2000, 85, 768.
(46) Modig, K.; Liepinsh, E.; Otting, G.; Halle, B. J. Am. Chem. Soc. 2004, 102, 102

(47) Hertz, H. Nuclear Magnetic Relaxation Spectroscopy. In Water: A Comprehensive Treatise; Plenum Press: New York, 1973; Vol. 3. 